\title{
Implantação do Sistema de Procedimentos de Alta Complexidade em Neurocirurgia. Resultados
}

\author{
Ericson Sfreddo ${ }^{1}$
}

Serviço de Neurocirurgia do Hospital Municipal de Novo Hamburgo, RS, Brasil

\section{RESUMO}

Objetivo: Demonstrar a maneira como foi implementado o Serviço de Alta Complexidade em Neurocirurgia num município da região metropolitana de Porto Alegre e seus resultados benéficos à população, aos neurocirurgiões e à Instituição Hospitalar. Material e método: São demonstrados os argumentos que o grupo de neurocirurgiões adotou para convencer a direção do Hospital Municipal de Novo Hamburgo (HMNH) a investir na especialidade antes mesmo de ter noção real das vantagens e dos lucros que adviriam. Resultados: Foi constatado que havia uma evasão mensal de aproximadamente 30 pacientes dos municípios que compunham a região para outro centro de referência ("Hospital Referência"). Essa evasão propiciaria um rendimento em torno de $R \$ 57$ mil. Foi demonstrado que o "Hospital Referência" havia saltado de um faturamento de pouco mais de $R \$ 100$ mil para aproximadamente $R \$ 500$ mil após a implantação do Sipac-Neuro, e que a média de valores das autorizações de internações hospitalares $(A I H)$ desse hospital era de $R \$ 1.056,12$ contra $R \$ 382,84$ do HMNH. A seguir, descrevem-se os passos que foram necessários para o credenciamento junto ao Ministério da Saúde, a implantação do serviço e os futuros investimentos que se pretende realizar em função dos novos aportes financeiros proporcionados pela Alta Complexidade. Os resultados financeiros iniciais após o credenciamento foram significativos. Conclusão: A organização e demonstração real, com exemplos de outras instituições, são instrumentos úteis e facilitadores para o convencimento das direções de hospitais que ainda são céticos quanto às vantagens proporcionadas pelo Sipac-Neuro.

\section{PALAVRA-CHAVE}

Administração hospitalar.

\section{ABSTRACT}

Implementation of high complexity service in a city hospital of metropolitan region of Porto Alegre city, Brazil

Objective: To present how a High Complexity Neurosurgical Service (accredited by the Ministry of Health denominated Sipac - Neuro) was implemented in a public hospital of the metropolitan region of the city of Porto Alegre, Brazil. Material and method: The arguments used by the group of neurosurgeons that convinced the direction of the Hospital Municipal de Novo Hamburgo (HMNH) to invest on neurosurgery, before knowing the advantages and profit that would be generated is presented. Result: It was verified that there was an monthly evasion rate of approximately 30 patients from the regions covered by HMNH to other reference center ("Reference" hospital) during the year of 2001. This evasion would have yielded around $R \$ 57,000$. It was demonstrated that the "Reference" hospital leaped from an income of around $R \$ 100,000$ to around $R \$ 500,000$ after the implementation of the Sipac-Neuro and that the average cost of Authorization for Hospitalization $(\mathrm{AlH})$ at that hospital was $R \$ 1,056.12$ compared to $R \$ 382.84$ at $\mathrm{HMNH}$. The method of accreditation with the Ministry of Health is described herein, as well as the implementation of the Service and the future investments that are to be made as a result of the new financial resources as a result of this accreditation for high complexity. Conclusion: It is concluded that the organization and actual demonstration by means of the examples of other institutions is a useful and helpful tool to convince hospital administrations who remain skeptical as to the advantages obtained with a Sipac-Neuro.

\section{KEY WORD}

Hospital administration. 


\section{Introdução}

$\mathrm{Na}$ atual conjuntura econômica do país, o médico tem se defrontado com uma situação em que, muitas vezes, se vê obrigado a dedicar muitas horas do seu trabalho com assuntos que não dizem respeito diretamente ao atendimento em enfermaria, rotinas de bloco cirúrgico, consultório etc.

Graças ao esforço de várias diretorias da Sociedade Brasileira de Neurocirurgia (SBN), em 1998 iniciou-se o reconhecimento do Sistema de Procedimentos de Alta Complexidade para Neurocirurgia, o Sipac-Neurocirurgia. Sem dúvida, a SBN foi a principal responsável para que o atendimento ao paciente neurocirúrgico, por meio do Sistema Único de Saúde (SUS), pudesse ser realizado de uma forma financeiramente interessante, e não apenas com o ideal científico de um legado deixado por saudosos mestres. A partir daquele ano, a neurocirurgia no Brasil tomou outro rumo.

Este artigo tem a pretensão de mostrar objetivamente que é possível despertar o interesse dos administradores hospitalares para a neurocirurgia; mostrar que a nossa especialidade é um caminho viável para, não somente ser auto-sustentável, mas também para alavancar as finanças de vários hospitais que atendem esse mercado.

\section{Material}

Foi revisada, passo a passo, a maneira que o grupo de neurocirurgiões adotou para convencer a direção do Hospital Municipal de Novo Hamburgo (HMNH) a investir na especialidade, antes mesmo de ter noção real das vantagens e dos lucros que adviriam:

1. Levantamento minucioso da demanda de casos neurocirúrgicos;

2. Levantamento das condições técnicas da instituição;

3. Apresentação, à Direção do HMNH, dos benefícios da implementação dos procedimentos de alta complexidade;

4. Busca do credenciamento Sipac-Neurocirurgia junto ao Ministério da Saúde;

5. Implantação do projeto.

\section{Método}

O primeiro passo seguido foi realizar um levantamento minucioso dos casos que evadiam do município. O HMNH é referência regional de dez municípios, o que abrange uma população de 476.427 habitantes. Possui plantão 24 horas de clínica médica, cirurgia geral, trau- matologia, obstetrícia, neonatologia e neurocirurgia, este último desde outubro de 1997. Como a remuneração para o plantonista é fixa e independe da quantidade de trabalho despendida, o neurocirurgião realizava apenas procedimentos de urgência. Os casos eletivos eram transferidos para a capital gaúcha, Porto Alegre. Baseado em dados do ano de 2001, constatou-se que, em média, 32 novos casos mensais eram enviados apenas para um dos hospitais de Porto Alegre que, caracteristicamente, não atende urgências (tabela 1). Desconsideraram-se os pacientes enviados para outros hospitais que são referências para traumatologia, os encaminhados diretamente das cidades da região sem passar pelo HMNH e os encaminhados pelo posto de saúde. Esses dados podem facilmente ser copilados do levantamento estatístico mensal, que é feito pelo Setor de Faturamento de todo hospital que trabalha com SUS ${ }^{1}$.

Tabela 1

\begin{tabular}{lc}
$\begin{array}{c}\text { Média mensal de pacientes neurocirúrgicos transferidos para } \\
\text { o "Hospital Referência", em Porto }\end{array}$ & Alegre, no ano de 2001 \\
\hline Cidade & $\mathbf{n}^{\mathbf{0}}$ pacientes \\
Novo Hamburgo & 18 \\
Campo Bom & 1 \\
Estância Velha & 3 \\
Portão & 3 \\
Sapiranga & 7 \\
Total & 32
\end{tabular}

Fonte: Setor de Faturamento do "Hospital Referência".

Essa evasão fazia com que o HMNH deixasse de faturar mensalmente, em média, o equivalente a R\$ 57.529,00 no mesmo período (tabela 2).

\begin{tabular}{lc}
\hline & Tabela 2 \\
\multicolumn{1}{c}{$\begin{array}{c}\text { Média de faturamento mensal do “Hospital } \\
\text { Referência” de pacientes neurocirúrgicos oriundos } \\
\text { da região do Vale dos Sinos no ano de 2001 }\end{array}$} \\
\hline Cidade & Valor em R\$ \\
Novo Hamburgo & $34.118,60$ \\
Campo Bom & $4.284,21$ \\
Estância Velha & $6.232,83$ \\
Portão & $2.306,17$ \\
Sapiranga & $10.587,19$ \\
Total & $57.529,00$ \\
\hline
\end{tabular}

Fonte: Setor de Faturamento do "Hospital Referência".

O segundo passo foi realizar um levantamento das condições técnicas da instituição em implantar os procedimentos de alta complexidade e adequação ao nível a ser implementado, de acordo com a portaria $\mathrm{GM} / \mathrm{MS} / \mathrm{N}^{\circ}$ 2920 de 9 de junho de 1998, publicada no DO n ${ }^{\circ} 111$ de 15 de junho de 1998 (nível I, nível II ou nível III) e portaria $\mathrm{n}^{\circ} 36$ de 4 de fevereiro de 1999 do Ministério da Saúde - Secretaria de Assistência da Saúde ${ }^{2,3}$.

Cabe ressaltar que não há diferença de valores entre os níveis citados para os respectivos procedimentos. 
A diferença está no tipo de procedimento que um hospital pode ou não fazer.

O terceiro passo foi mostrar à Direção do hospital os benefícios da capacitação para o Sipac-Neurocirurgia. $\mathrm{O}$ atendimento global do paciente que procura atendimento em um hospital é o que traz a credibilidade à instituição. A necessidade de freqüentes buscas de solução em outras instituições traz a incerteza do tratamento correto. Nesse sentido, a neurocirurgia destaca-se pela sua importância como especialidade já que, além da complexidade de seu atendimento, é provedora de AIH rentável.

Nada melhor do que demonstrar o desempenho de outros hospitais após a adoção dessa nova perspectiva e, fundamentalmente, na mudança de comportamento de todas as pessoas envolvidas nesse tipo de atendimento. Como o "Hospital Referência", aqui citado para comparação, é uma entidade privada que depende dos recursos do SUS, criou-se nesse nosocômio a necessidade de aproveitar-se, ao máximo, cada autorização de internação hospitalar para que se pudesse ter mais lucro com as mesmas despesas. Mais uma vez comprova-se a lei de que "a função faz o órgão". Isso fica claro no levantamento feito junto ao site do Datasus ${ }^{1}$ que demonstra claramente a diferença de valores pagos por AIH entre os dois hospitais (tabela 3).

Tabela 3

Média por AIH referente ao mês de novembro de 2001

\begin{tabular}{lccc}
\hline Hospital & AIH (n) & Valor total & Média por AIH \\
"Referência" & 476 & $502.711,63$ & $1.056,12$ \\
HMNH & 645 & $246.932,61$ & 382,84 \\
\hline
\end{tabular}

Fonte: DATASUS

A análise da tabela 3 levanta três hipóteses:

1. As AIH do HMNH são "fracas", isto é, são procedimentos que não remuneram tanto. Isso em parte é verdade, já que o HMNH atende emergência clínica e politraumatizados que são, sabidamente, atendimentos financeiramente deficitários; já o "Hospital Referência" atende apenas algumas poucas emergências clínicas;

2. As AIH do HMNH são mal faturadas;

3. Ambas.

Outro ponto fundamental foi mostrar a comparação entre o faturamento de um determinado hospital antes e depois do Sipac-Neurocirurgia (tabela 4).

\begin{tabular}{lcccc}
\hline \multicolumn{5}{c}{ Tabela 4 } \\
& $\begin{array}{c}\text { Faturamento do “Hospital Referencia” } \\
\text { antes e depois do Sipac-Neuro }\end{array}$ \\
\hline $\begin{array}{l}\text { Período } \\
\text { Antes do }\end{array}$ & Agosto & Setembro & Outubro & Novembro \\
$\begin{array}{l}\text { Sipac (98/99) } \\
\text { Depois do } \\
\text { Sipac (01/02) }\end{array}$ & $177.099,72$ & $129.880,84$ & $121.506,46$ & $95.520,45$ \\
\hline Fonte: DATASUS & $501.560,50$ & $428.198,20$ & $474.062,03$ & $502.711,63$ \\
& & & & \\
\end{tabular}

O quarto passo é a busca da autorização, junto ao Ministério da Saúde, para os procedimentos de alta complexidade. Para que isso ocorra, é necessário seguir os seguintes passos:

1. A unidade hospitalar encaminha seu pedido de credenciamento no Sistema de Procedimentos de Alta Complexidade para Neurocirurgia - Sipac, do Sistema Único de Saúde, ao município onde está localizado, preenchendo questionário específico fornecido pelo Ministério da Saúde;

2. A Secretaria de Saúde do município procederá à vistoria nos parâmetros estabelecidos e atribuirá o nível do serviço a ser implantado de acordo com as condições técnicas do hospital, que poderá ser I, II ou III. A seguir, encaminhará o pedido e o laudo à Secretaria de Saúde do Estado a que pertence;

3. A Comissão Bipartite da Secretaria de Saúde do Estado vistoria o hospital solicitante e aprova ou faz a correção quanto ao nível solicitado. A seguir, procederá aos cálculos dos adicionais financeiros, lançará em orçamento e remeterá à Secretaria de Assistência à Saúde do Ministério da Saúde;

4. O Ministério da Saúde analisará os requerimentos efetuados e os laudos de auditoria procedidos no hospital, concluindo pelo credenciamento ou não dentro do Sipac-Neurocirurgia. Os hospitais credenciados terão publicidade desse fato por portaria assinada pelo ministro da Saúde.

O quinto passo é a implementação do serviço. Isso depende da boa vontade e do trabalho auspiciosos que cada um deve desprender. A manutenção do serviço Sipac-Neurocirurgia depende, não só do trabalho, mas da vigilância e exigência permanentes do neurocirurgião tanto no Setor de Faturamento quanto no de Administração do hospital, sempre buscando melhorias nas condições técnicas para, assim, tornar o atendimento neurocirúrgico o mais próximo do ideal possível.

\section{Resultado}

A direção do HMNH aceitou a proposta e resolveu apostar na iniciativa do grupo. Para tanto, foram tomadas algumas providências:

1. A primeira medida foi a compra de material cirúrgico para as cirurgias eletivas (já havia material disponível para cirurgias de urgência). Esse material foi composto de uma caixa de craniotomia, uma caixa de laminectomia, material de microcirurgia, microscópio e demais acessórios, tais como afastadores cerebrais, afastadores de corpos vertebrais, coagulador bipolar, coagulador unipolar etc. Esse investimento foi de R $\$ 150$ mil; 
2. Reforma de uma unidade para nove leitos destinados a internações eletivas para tratamentos chamados de "conservadores" (clínicos) em neurocirurgia. Cabe salientar que já há outra ala com 30 leitos para pacientes cirúrgicos de todas as áreas (traumatologia, cirurgia geral e neurocirurgia);

3. Ampliação dos leitos de UTI de seis para oito e, num futuro próximo, para dez leitos (já aprovado por vistoria e publicado no Diário Oficial da União);

4. Melhoramento no Setor de Faturamento, no qual havia muitos erros na cobrança dos códigos de SSM. Para a correção dessa distorção, foi contratada, pelo HMNH, uma equipe especializada em faturamento. Com isso, houve uma melhora substancial nos valores médios das AIH. Não é possível determinar o quanto isso foi significativo por haver muitas variáveis que poderiam ser responsáveis por tal mudança, mas é notório que não foram mais deixados para trás cobranças de diárias de acompanhantes, diárias de UTI, mudança de códigos, uso de códigos de cirurgias múltiplas e procedimentos seqüenciais em neurocirurgia e, principalmente, maior participação dos médicos assistentes no preenchimento das documentações. Este último fator talvez seja o mais importante, e é nesse item que reside um importante aliado do hospital: o médico que recebe pelo que faz (produtividade) tem interesse especial em preencher corretamente as documentações, aumentar a rotatividade dos pacientes internados e escolher os códigos SSM de melhor valor.

No gráfico 1, podemos comparar os valores líquidos recebidos pelo HMNH entre 2002 - quando existia a alta complexidade, porém os médicos recebiam valores fixos pelo plantão, portanto, só operavam cirurgias de urgência - e 2003, quando foi implantado o Serviço de Neurocirurgia e os médicos passaram a receber os valores das AIH.

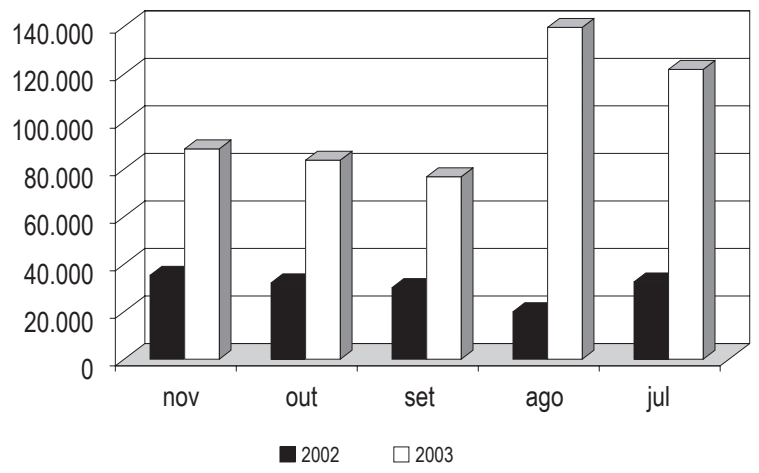

Gráfico 1 - Valores recebidos pelo HMNH em 2002 (antes do Sipac-Neuro) e 2003 (depois do Sipac-Neuro)
Além disso, a média de valores recebidos pelas $\mathrm{AIH}$ de todo o hospital teve um aumento significativo de aproximadamente $46 \%$, enquanto a média dos valores recebidos por AIH só da Alta Complexidade passou de $\mathrm{R} \$ 813,71$ para $\mathrm{R} \$ 1.554,73$, conforme podemos constatar na tabela 5 .

\begin{tabular}{lccc}
\hline \multicolumn{4}{c}{ Tabela 5} \\
\multicolumn{4}{c}{$\begin{array}{c}\text { Média de valores recebidos por AIH do HMNH antes } \boldsymbol{e} \\
\text { depois da implementação do Serviço de Neurocirurgia }\end{array}$} \\
\hline Período & AIH (N) & Valor total & Média por AIH \\
Antes & 645 & $246.932,61$ & 382,84 \\
Depois & 756 & $424.888,27$ & 561,77 \\
Neuro antes & 51 & $41.499,40$ & 813,71 \\
Neuro depois & 66 & $102.612,18$ & $1.554,73$ \\
\hline
\end{tabular}

Fonte: DATASUS ${ }^{1}$

\section{Conclusão}

Fica claro, diante do exposto, que é muito lucrativo para a instituição investir em neurocirurgia, tanto do ponto de vista econômico como da credibilidade que o hospital adquire perante a sociedade, que passa a ter atendimento mais qualificado e prestativo e evita o incômodo e arriscado transporte a um centro de referência. Com todos esses aspectos, a neurocirurgia pode ser uma especialidade auto-sustentável, mesmo atendendo pacientes do SUS, e melhorar o desempenho financeiro da instituição hospitalar, no que tange as outras especialidades.

O neurocirurgião não deve deter-se apenas ao atendimento médico, mas buscar sempre as melhores condições de trabalho, lembrando sempre que conhecer a burocracia em detalhes faz parte do procedimento médico ao atender qualquer paciente, seja do SUS, seja particular ou de outros convênios.

\section{Referências}

1. DATASUS, Ministério da Saúde, República Federativa do Brasil. Consulta ao Banco de Dados - BDAIH [Serial online] 2005. Disponível em URL: http://bdaih.datasus.gov. $\mathrm{br} / \mathrm{scripts/menu.asp.}$

2. DIÁRIO OFICIAL DA UNIÃO (111): Portaria GM/MS/Nº 2920 15 de junho de 1998.

3. DIÁRIO OFICIAL DA UNIÃO: Ministério da Saúde - Secretaria de Assistência da Saúde. Portaria GM/MS/Nº 364 de fevereiro de 1999.

Original recebido em outubro de 2006

Aceito para publicação em março de 2007

\author{
Endereço para correspondência \\ Ericson Sfreddo \\ Travessa Jundiai, $2.200 / 1.103$ \\ 90520-270 - Porto Alegre, RS \\ E-mail: ericsonsfreddo@gmail.com
}

